\title{
Measurement of the Cam Spacing on Camshaft by Binocular Vision
}

\author{
Yueqian $\mathrm{Hou}^{1, *}$, Qingchang $\mathrm{Tan}^{2}$, Qinghua $\mathrm{Li}^{1}$, Yumei Song ${ }^{1,}$ and Guannan $\mathrm{Li}^{3}$ \\ ${ }^{1}$ Mechanical and Vehicle Engineering College, Changchun University, Changchun \\ 130022, China \\ ${ }^{2}$ The College of Science and Mechanical Engineering, Jilin University, Changchun \\ 130025, China \\ ${ }^{3}$ Changchun Institute of Optics, Fine Mechanics and Physics, Chinese Academy of \\ Sciences, Changchun 130022, China \\ 909335779@qq.com
}

\begin{abstract}
This paper proposed a measurement method of the cam spacing of amshaft based on binocular vision. Firstly, internal and external parameters of the camero were calibrated by the improved calibration. Secondly, the objective function is established by the distance of pixel coordinates and their corresponding epipolar lines, and the fundamental matrix is obtained by optimizing the objective function. Finally, the translation vector and the rotation matrix are determined by the singular value deconposition. Using the results of calibration, a method of measuring can spacing of counshaft is presented. In the experiment condition, cam spacing of camshaft is medsured. Experiment result shows that accuracy of the proposed method could meet the requirements of monitoring the cam spacing of combined type camshaft.
\end{abstract}

\section{Introduction}

Camshaft is one of the important components in engine. Modern technology mostly adopted a combination of hollow structure. Process requires the precision is smaller than $\pm 0.3 \mathrm{~mm}$ [1-2]. Because there is a phase difference of every cam along the circumference of the cam, the distance between two cams is a 3-dimensional size.

In the past, many researchers have developed algorithms for the 3-dimensional reconstruction by binocalar vision. K. Zhang [3] explores a model of the binocular vision system focused on 3D reeonstruction and describes an improved genetic algorithm aimed at estimating canera system parameters. In order to enhance the calibration accuracy, many corners should be treated as feature points. W. Sun [4] presents a study investigating the effects of training data quantity, pixel coordinate noise on binocular vision accuracy. $\mathrm{H}$. $\mathrm{H}$. Cui [5] discusses an improved method for an accurate 3-dimensional measurement. The system accuracy is improved considering the nonlinear measurement error. An aecurate phase-height mapping algorithm is proposed by $\mathrm{Z}$. W. Li [6] to improve the performance of the structured light system with digital fringe projection. By means of a training network, the relationship between the 2D image coordinates and the 3D object coordinates can be achieved. However, their experiments involve fixed system structure parameters and provide a synthetic evaluation on flexible binocular system parameters to verify the accuracy results.

This article proposed a measurement method of can spacing by binocular vision. This work can meet the requirement of practical measurement, and realize the automatic detection.

${ }^{*}$ Corresponding Author 


\subsection{Binocular Vision Model}

Binocular vision model could be expressed based on the perspective transformation model, which is shown in Figure 1.

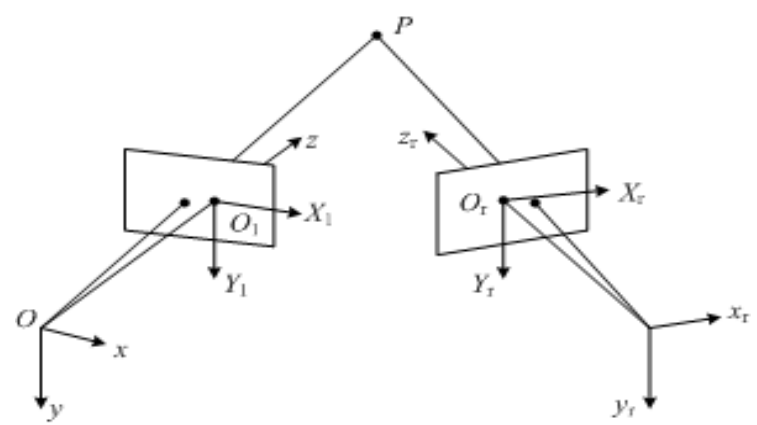

Figure 1. Binocular Vision Model

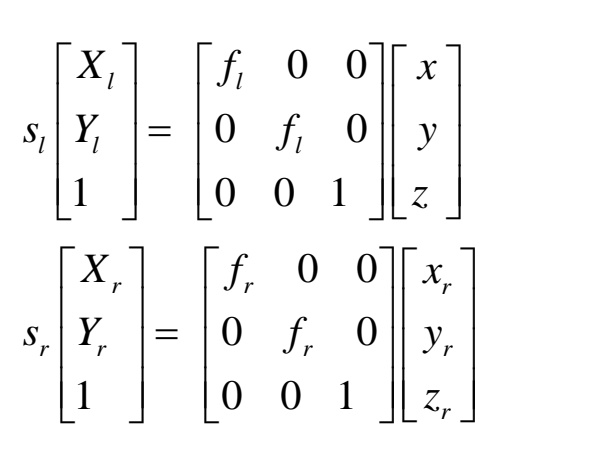

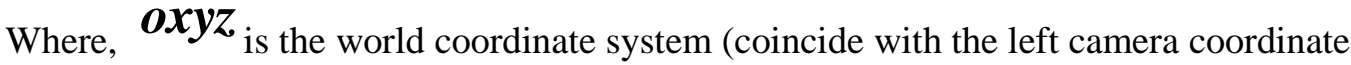
system), $\boldsymbol{o}_{r} \boldsymbol{x}_{r} \boldsymbol{y}_{r} \boldsymbol{z}_{r}$ is the right camera coordinate system, $\boldsymbol{O}_{l} \boldsymbol{X}_{l} \boldsymbol{Y}_{l}, \boldsymbol{O}_{r} \boldsymbol{X}_{r} \boldsymbol{Y}_{r}$ are the left and right cameras' inge coordinate systems. $f_{l}, f_{r}$ are effective focal lengths of the left and right cameras. $\boldsymbol{P}_{\text {is }}$ the measured point in the public view. Relationship between the right camera coordinate system and the the world coordinate system can be expressed as:
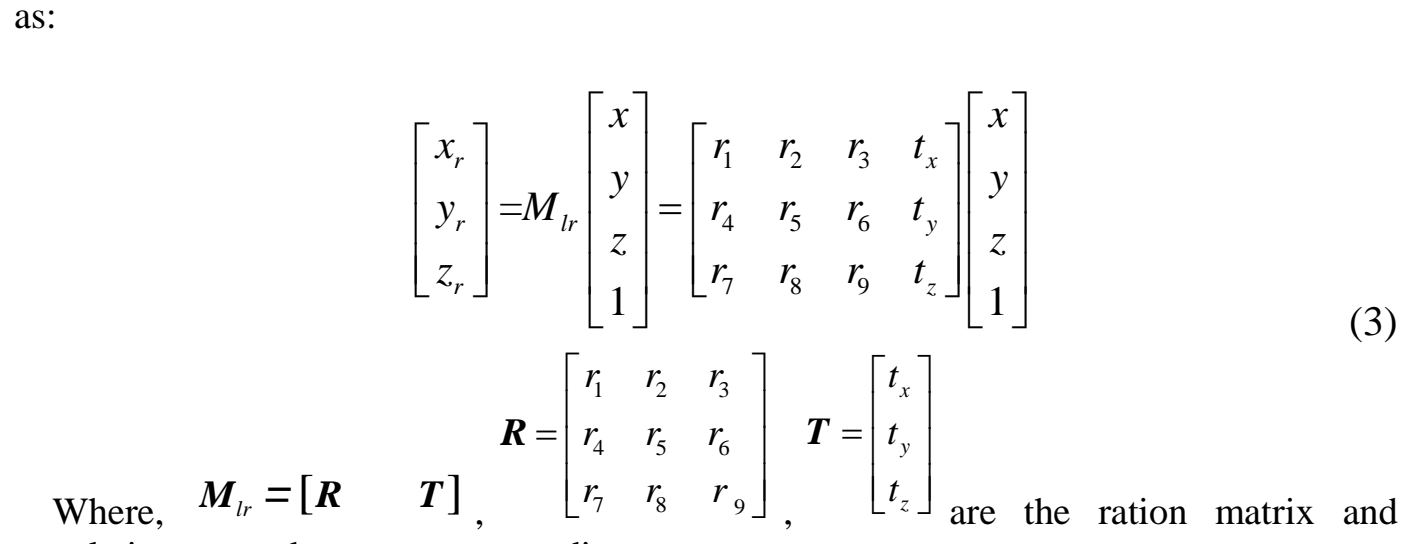
translation vector between two coordinate systems.

From Eq. (1), (2) and (3), coordinates of $\boldsymbol{P}$ in the 3 dimensional world coordinate system can be obtained: 


$$
\left\{\begin{array}{c}
x=z X_{1} / f_{1} \\
y=z Y_{1} / f_{1} \\
z=\frac{f_{1}\left(f_{r} t_{x}-X_{r} t_{z}\right)}{X_{r}\left(r_{7} X_{1}+r_{8} Y_{1}+f_{1} r_{9}\right)-f_{r}\left(r_{1} X_{1}+r_{2} Y_{1}+f_{1} r_{3}\right)} \\
=\frac{f_{1}\left(f_{r} t_{y}-Y_{r} t_{z}\right)}{Y_{r}\left(r_{7} X_{1}+r_{8} Y_{1}+f_{1} r_{9}\right)-f_{r}\left(r_{4} X_{1}+r_{5} Y_{1}+f_{1} r_{6}\right)}
\end{array}\right.
$$

\section{Key Techniques of Binocular Vision}

From Eq.(4), some work has to be carried out in the visual measurement:(1) the calibration of effective focal length $f_{l}, f_{r}$; (2) relationship between twe camera coordinate system; (3) matching of corresponding points in the left and right image.

\subsection{Calibration of Effective Focal Length}

The calibration of CCD camera can be divided to the calculating of interior camera parameters and exterior camera parameters, which directly affect the accuracy of measurement. A large number of research work have been carned out by scientists [7-8]. An improved method [9] is used to calibrate the effective focal length.

\subsection{Matching of Corresponding Points and Fundamental Matrix}

Polar geometric constraint is one of the mostamportant constraints in the matching of corresponding points in the left and right images, which can be expressed by fundamental matrix. The ration matrix and translation vector between two cameras can be obtained by the decomposing of fundamental natrix. As a result, the calibration of fundamental matrix is the most important technology in the binocular vision measurement.

The fundamental matrix can be writtenas [10]:

$$
\boldsymbol{F}=\boldsymbol{A}_{r}^{-T} \boldsymbol{S} \boldsymbol{R} \boldsymbol{A}_{l}^{-1}
$$

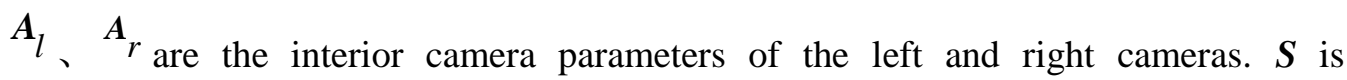
anti-symmetric matrix, which can be defined by the translation vector between two cameras:

$$
\boldsymbol{S}=[t]_{\times}=\left[\begin{array}{ccc}
0 & -t_{z} & t_{y} \\
t_{z} & 0 & -t_{x} \\
-t_{y} & t_{x} & 0
\end{array}\right]
$$

Let $\boldsymbol{E}=\boldsymbol{S R}=\boldsymbol{A}_{r}^{T} \boldsymbol{F} \boldsymbol{A}_{l}$

is the essential matrix. The ration matrix and translation vecor between two cameras can be calculated by singular value decomposition of $\boldsymbol{E}$.

$$
\boldsymbol{R}=\boldsymbol{U}\left[\begin{array}{ccc}
0 & -1 & 0 \\
1 & 0 & 0 \\
0 & 0 & 1
\end{array}\right] \boldsymbol{V}
$$




$$
\boldsymbol{T}=k \boldsymbol{U}\left[\begin{array}{l}
0 \\
0 \\
1
\end{array}\right]
$$

\subsection{Calculation of Fundamental Matrix}

There were a lot of methods to calculate fundamental matrix. Armangue $\mathrm{X}$ divided these methods into three types: linear method, iterative method and robust method. 8 points method is the most representative method in linear method. It is sensitive to noise, and the robustness is poor. So it is difficult to use in practice. Although the accuracy of the iterative method has been improved, the calculation time is longer. At the same time, the problem of noise is not solved. Robust method's accuracy is improved, and the problem of noise is solved. But the algorithm is too complicated, which is not easy to achieve

Because the pixel coordinates extracted from model plane have the feature of high accuracy and good stability, they were used to obtain a precise and stable fundamental matrix. Based on the relationship of polar geometric constraint, the distance between the pixel point and its corresponding polar line is used as thê objectiye function of optimization to calculate the fundamental matrix.

Let $\mathrm{I}^{\prime}=\left(l_{1}^{\prime}, l_{2}^{\prime}, l_{3}^{\prime}\right)=\mathrm{Fm}, \mathrm{I}=\left(l_{1}, l_{2}, l_{3}\right)=\mathbf{m}^{\top T} \mathrm{~F}$ distance between the pixel point and its corresponding polar line can be expressed as:

$$
d\left(m^{\prime}, l^{\prime}\right)=\frac{\left|\hat{\mathrm{m}}^{\prime} \mathrm{T}\right|}{\sqrt{\left(l_{1}^{\prime}\right)^{2}+\left(l^{\prime}\right)^{2}}}
$$

Where, $d\left(m^{\prime}, l^{\prime}\right)$ is the distance between point in the image of left camera and its corresponding polar line in the image of right camera; $d(m, l)$ is the distance between point in the image of right canera and its corresponding polar line in the image of left camera; $m$ is the pixel coordinates of point in the left camera; $m$ is the pixel coordinates of point in the right camere

As a result, theobjective function can be defined as:

$$
\begin{aligned}
& \text { Wherck }=\sqrt{\left(l_{1}^{\prime}\right)^{2}+\left(l_{2}^{\prime}\right)^{2}}, k=\sqrt{\left(l_{1}\right)^{2}+\left(l_{2}\right)^{2}} \text {. } \\
& \text { 3. Measurement of Cam Spacing on Assembled Camshaft }
\end{aligned}
$$

\section{Measurement of Cam Spacing on Assembled Camshaft}

\subsection{Calibration of Parameter}

In the laboratory, two JAI CCD camera with the resolution of $1392 \times 1040$ pixel was used to establish a binocular vision measurement system. The precious of model plane is $\pm 1 \mu \mathrm{m}$. The measured camshaft is shown in Figure 2 . 


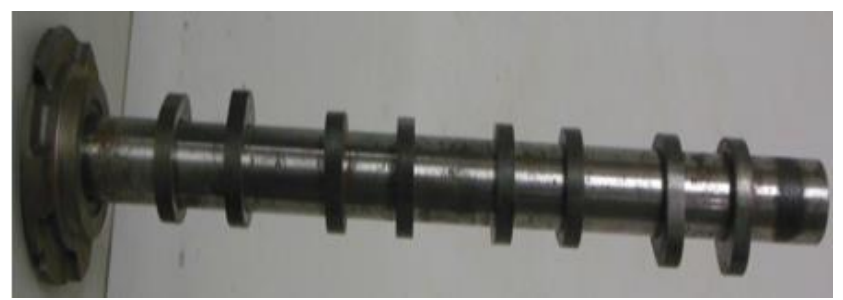

Figure 2. Measured Camshaft

The effective focal lengths of two cameras are obtained by the method proposed in $^{[11]}$ :

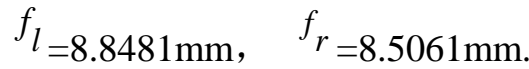

Using the corner points on the model plane, the fundamental matrix is fitted:

$$
\boldsymbol{F}=\left[\begin{array}{lll}
-1.4999 \mathrm{E}-5 & -1.2035 \mathrm{E}-4 & 0.0324 \\
1.1852 \mathrm{E}-4 & -3.7553 \mathrm{E}-5 & -0.0721 \\
-0.0073 & 0.0720 & -8.5615
\end{array}\right]
$$

From the fundamental matrix, the relationships between the points and their corresponding polar lines are shown in Figure 3:

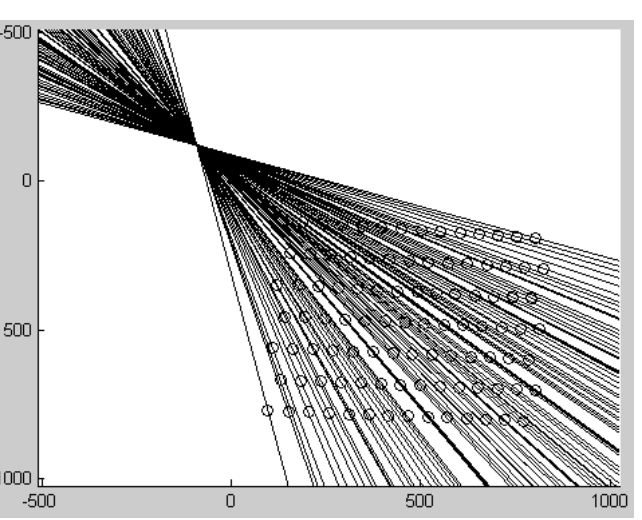

Figure 3. Relationships Between the Points and their Corresponding Polar

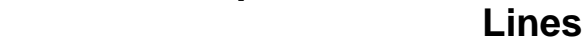

Decomposing the fundamental matrix, the ration matrix and translation vector between two cameras could be obtained:

$$
R=\left[\begin{array}{lll}
-0.9757 & 0.2184 & -0.0163 \\
-0.2123 & -0.9613 & -0.1753 \\
-0.0540 & -0.1676 & 0.98436
\end{array}\right] \quad T=\left[\begin{array}{c}
-2.8002 \\
-8.5672 \\
89.1906
\end{array}\right]
$$

The fundamental matrix provides a constraint for the matching of corresponding points. The edge of the cam can be taken as the other constraint, which is shown in Figure4. 


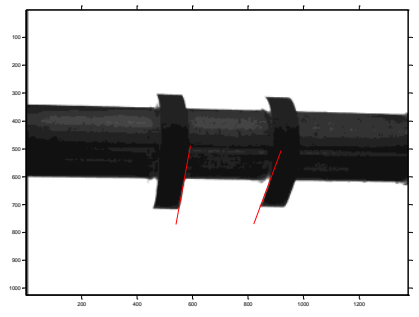

a. Left Camera

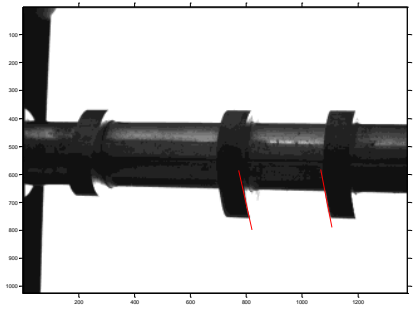

b. Right Camera

Figure 4. Image for Measurement

\subsection{Experimental Result}

From the corresponding edge points, the 3-dimensional points on the cam edge Was reconstructed, which is shown in Figure 5. By the linear fitting and calculating of distance between straight lines, the cam spacing was obtained.

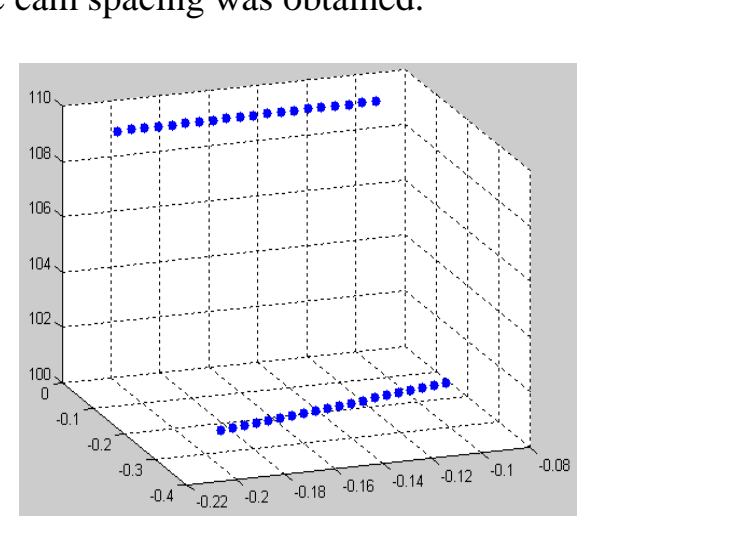

Figure 5 -Result of 3 -Dimensional Reconstruction

The result is contrasted to the measurement by vernier caliper, the results repeated 5 times for each cam spacing is shown in Table 1.

Table 1. Result of Measuring Cam Spacing ( $\mathrm{mm}$ )

\begin{tabular}{|c|c|c|c|c|c|c|c|}
\hline & & 2 & 3 & 4 & 5 & Mean & Standard deviation \\
\hline Proposed method & 30.0 & 30.0 & 30.1 & 30.0 & 30.1 & 30.0 & 0.0 \\
& 941 & 894 & 000 & 899 & 201 & 987 & 127 \\
\hline Vernier caliper & 30.0 & 30.0 & 30.0 & 30.0 & 30.0 & 30.0 & 0.0 \\
& 31 & 26 & 32 & 43 & 50 & 364 & 0987 \\
\hline
\end{tabular}

From the measurement result, the error of the proposed method is smaller than $\pm 0.1 \mathrm{~mm}$, which can meet the requirement of practical measurement.

\section{Conclusions}

A vision measurement method is proposed for measuring cam spacing on assembled camshaft. This method can meet the requirement of practical measurement, which will reduce the labor intensity of workers. 


\section{Acknowledgments}

This work supported by the Project supported by the Maor International Joint Research Program of China (Grant No. 2013DFA71120), and Supported by Harbin Natural Science Funds for Distinguished Young Scholar (Grant No. 2015RAYXJ001).

\section{References}

[1] C. Hughes, C. Mitts, F. Jacquelin and M. Wiseman, "Heavy duty truck cooling system design-using co-simulation", SAE 2001-01-1707.

[2] Aricio, J. H. Johnson and J. Ajey, "The vehicle engine cooling system simulation part1-model development" SAE 1999-01-0240.

[3] K. Zhang, B. Xu, L. Tang and H. Shi, "Modeling of binocular vision system for 3D reconstruction with improved genetic algorithms", Int. Journal Adv. Manuf. Technol., vol. 29, (2006), pp. 722-728.

[4] W. Sun and J. R. Cooperstock, "An empirical evaluation of factors influencing camera calibration accuracy using three publicly available techniques", Mach. Vision Appl., vol. 17, (2006), pp. 51-67.

[5] H. H. Cui, W. H. Liao, X. S. Cheng, N. Dai and T. R. Yuan, "A three-step system calibration procedure with error compensation for 3D shape measurement", Chinese Opt. Lett., vol. 8, (2010), pp. 3

[6] Z. W. Li, Y. S. Shi, C. J. Wang, D. H. Qin, and K. Huang, "Complex object 3D measurement based on phase-shifting and a neural network", Opt. Comm., vol. 282, (2009), pp. 2699-2706.

[7] Z. Zhang, "Determining the epipolar geometry and its uncertainty: A review", Int. Journal Computer vision, vol. 27, no. 2, (1998), pp. 161-195.

[8] R. Y. Tsai, "A versatile camera calibration technique for high accuracy 3D machine vision metrology using off-the-shelf TV cameras and lenses", IEEE J Robotics Automat, RA-3, no. 4, (1987), pp. 323-344.

[9] Z.Y. Zhang, "A flexible new technique for camera calibration", IEEE Transactions on pattern Analysis and Machine Intelligence, vol. 22, no. 11, (2000), pp. 1330-1334.

[10] H. Yueqian, T. Qingchang, W. Kai and S. Qiucheng, "An improved method for calculating extrinsic and intrinsic parameters of camera", Computer applications and software, vol. 27, no. 10, (2010), pp. 114-116.

[11] S. Qiucheng, "Measurement of shaft diameters from machine vision", Jilin University, (2010).

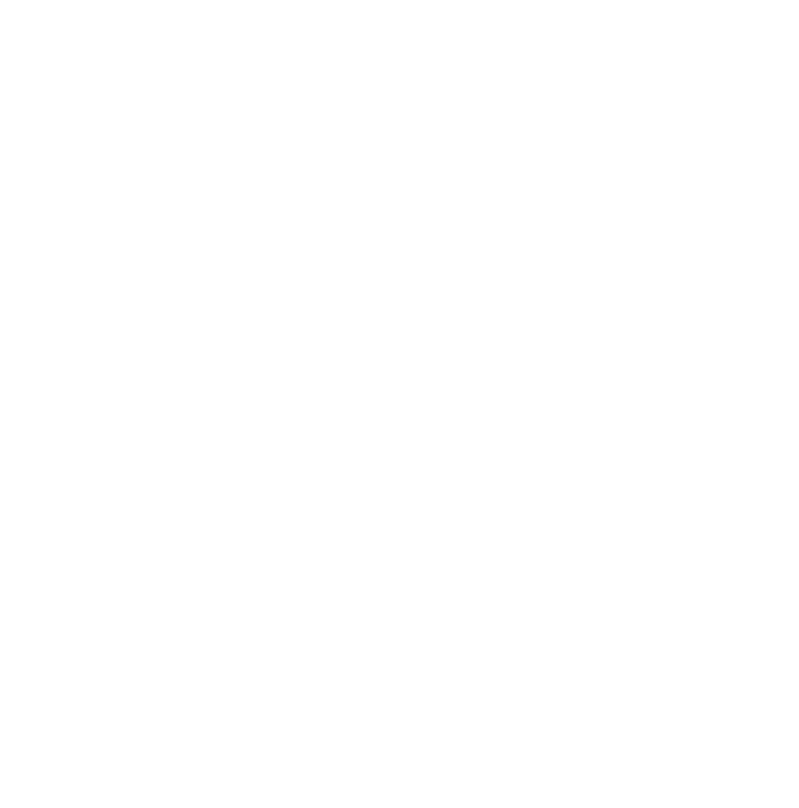


International Journal of Multimedia and Ubiquitous Engineering

Vol.11, No.9 (2016)

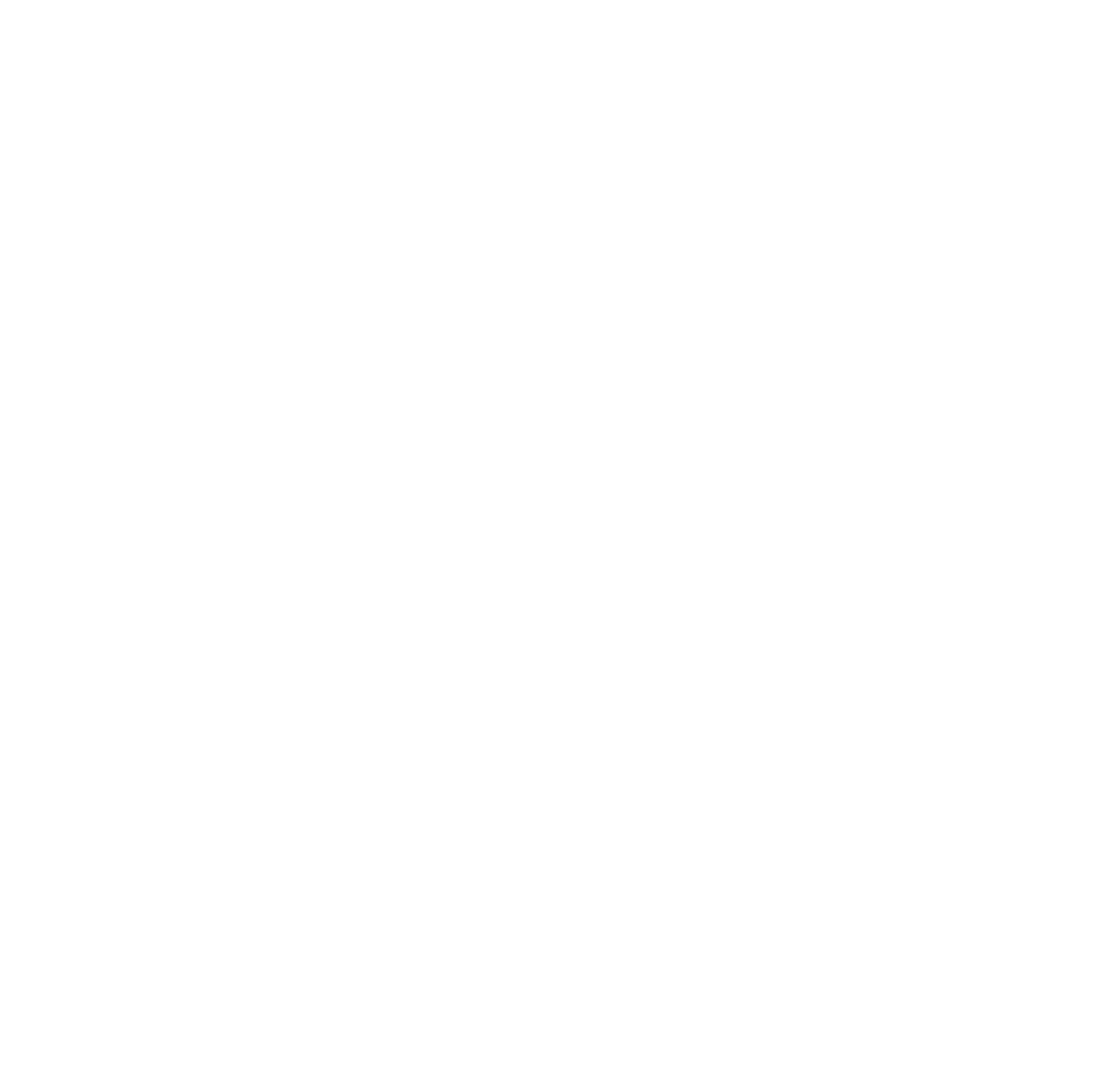

